\title{
Neural Network-Based Residual Capacity Indicator for Nickel-Metal Hydride Batteries in Electric Vehicles
}

\author{
W. X. Shen, Member, IEEE, K. T. Chau, Senior Member, IEEE, C. C. Chan, Fellow, IEEE, and \\ Edward W. C. Lo, Member, IEEE
}

\begin{abstract}
This paper presents a new estimation approach for the battery residual capacity (BRC) indicator in electric vehicles (EVs). The key of this approach is to model the EV battery by using a neural network (NN) with a newly defined output and newly proposed inputs. The inputs are the discharged and regenerative capacity distribution and the temperature. The output is the state of available capacity (SOAC) which represents the BRC. Various SOACs of the nickel-metal hydride (Ni-MH) battery are experimentally investigated under different EV discharge current profiles and temperatures. The corresponding data are recorded to train and verify the proposed NN. The results indicate that the NN can provide an accurate and effective estimation of the BRC. Moreover, this NN can be easily implemented as the BRC indicator or estimator for EVs by using a low-cost microcontroller.
\end{abstract}

Index Terms-Electric vehicle (EV), neural network (NN), nickel-metal hydride (Ni-MH), battery residual capacity (BPC) indicator.

\section{INTRODUCTION}

I N RESPONSE to the ever-increasing concerns on environmental protection and energy conservation, research and development of various technologies for electric vehicles (EVs) are being actively conducted [1]-[8]. Among those EV technologies, the battery technology is the key to possible commercialization and popularization of EVs. In the present status of battery technology, the range of EVs per charge is only about $100 \mathrm{~km}$ for urban driving which is much lower than that of gasoline vehicles. So, the corresponding battery capacity needs to be fully utilized, and hence, the estimation accuracy of its indicator is highly essential, aiming to achieve an error less than $3 \%$.

Many types of batteries can be selected as energy sources in EVs. Nevertheless, recent development of EV batteries is mainly focused on the lead-acid, nickel-metal hydride $(\mathrm{Ni}-\mathrm{MH})$, and lithium-ion (Li-ion) batteries [9]-[14]. Due to mature technol-

Manuscript received September 20, 2001; revised June 4, 2003, June 17, 2004, November 10, 2004, January 14, 2005, February 26, 2005. This work was supported by the Committee on Research and Conference Grants of The University of Hong Kong, and the Research Grants Council of Hong Kong Special Administrative Region, China. The review of this paper was coordinated by Dr. M. Shahgir Ahmed.

W.X. Shen was with the Department of Electrical and Electronic Engineering, The University of Hong Kong, Pokfulam Road, Hong Kong. He is now with the School of Engineering, Monash University Malaysia, Malaysia (e-mail: shen.wei.xiang@eng.monash.edu.my).

K. T. Chau, C. C. Chan, and E. W. C. Lo are with the Department of Electrical and Electronic Engineering, The University of Hong Kong, Pokfulam Road, Hong Kong (e-mail: ktchau@eee.hku.hk; ccchan@eee.hku.hk; eewclo@polyu.edu.hk).

Digital Object Identifier 10.1109/TVT.2005.853448
TABLE I

Comparison of BACs Under DifFERENT Discharge CURRENT PRofILES

\begin{tabular}{cc}
\hline Discharge current profiles & BAC (Ah) \\
\hline 15 A for first $3600 \mathrm{~s}, 45 \mathrm{~A}$ for next $1200 \mathrm{~s}, 90 \mathrm{~A}$ for last $207 \mathrm{~s}$ & 35.2 \\
$90 \mathrm{~A}$ for first $207 \mathrm{~s}, 15 \mathrm{~A}$ for next $3600 \mathrm{~s}, 45 \mathrm{~A}$ for last $1447 \mathrm{~s}$ & 38.2 \\
$45 \mathrm{~A}$ for first $1447 \mathrm{~s}, 90 \mathrm{~A}$ for next $207 \mathrm{~s}, 15 \mathrm{~A}$ for last $5211 \mathrm{~s}$ & 44.9 \\
\hline
\end{tabular}

ogy and low cost, the lead-acid battery has been widely accepted in EVs. But, it suffers from low-specific energy and short-cycle life. In contrast, the Li-ion battery offers high-specific energy and long-cycle life. However, its high cost limits its practical application to EVs. Meanwhile, the Ni-MH battery has higher specific energy and longer cycle life than the lead-acid battery as well as lower cost than the Li-ion battery. It can also offer the advantages of environmental friendliness, rapid charge capability and maintenance-free operation. Thus, the Ni-MH battery is superseding the lead-acid battery as the most popular energy source for modern EVs [11]. It should be noted that the nickelcadmium (Ni-Cd) battery can offer characteristics, such as the nominal voltage, energy density, power density, and cycle life, similar to the Ni-MH battery. However, the scarcity of metallic cadmium is impeding the Ni-Cd battery from becoming a major energy source in modern EVs [14].

The Ni-MH battery converts its electrical energy into chemical energy based on the following reversible reaction [15]:

$$
\mathrm{MH}+\mathrm{NiOOH} \leftrightarrow \mathrm{M}+\mathrm{Ni}(\mathrm{OH})_{2} .
$$

Its active materials are hydrogen in the form of a metal hydride for the negative electrode and nickel oxyhydroxide for the positive electrode. An aqueous solution of potassium hydroxide works as the electrolyte. When the battery is discharged, the metal hydride is oxidized to form metal alloy and the nickel oxyhydroxide is reduced to nickel hydroxide. When the battery is charged, the reverse reactions occur. Both the discharge current profile and the temperature significantly affect these chemical reactions.

Due to the dependence of the EV driving range on the battery available capacity (BAC), the influences of discharge current profile and temperature on the BAC are experimentally investigated. The adopted Ni-MH battery has the rated capacity of 45 Ah at three-hour discharge rate, cycle life of 1500 , cost of USD $\$ 1100$, nominal voltage of $24 \mathrm{~V}$, and cutoff voltage of $20 \mathrm{~V}$. In order to investigate the influence of discharge current profile on the BAC, three different discharge current profiles are selected to discharge the battery under the same temperature of $25^{\circ} \mathrm{C}$. As 
TABLE II

COMPARISON OF BACs UNDER DIFFERENT TEMPERATURES

\begin{tabular}{lcccc}
\hline & \multicolumn{4}{c}{ BAC (Ah) } \\
& $15 \mathrm{~A}$ & $45 \mathrm{~A}$ & $90 \mathrm{~A}$ & $112.5 \mathrm{~A}$ \\
\hline $10^{\circ} \mathrm{C}$ & 44.0 & 43.5 & 42.1 & 41.5 \\
$25^{\circ} \mathrm{C}$ & 45.2 & 44.2 & 43.2 & 42.8 \\
$40^{\circ} \mathrm{C}$ & 46.9 & 45.4 & 44.9 & 44.1 \\
\hline
\end{tabular}

shown in Table I, the resulting BACs under these three discharge current profiles are very different. On the other hand, in order to investigate the influence of temperature on the BAC, four constant discharge currents are used to discharge the battery under the temperatures of $10^{\circ} \mathrm{C}, 25^{\circ} \mathrm{C}$, and $40^{\circ} \mathrm{C}$. As shown in Table II, the resulting BACs significantly vary with temperatures. Thus, both the discharge current profile and temperature should be taken into account during the development of battery residual capacity (BRC) indicators for Ni-MH batteries. Also, since the driving style directly relates to the discharge current profile of EVs, it definitely influences the corresponding BRC, and hence, the driving range.

Within the last three decades, the BRC estimation for the lead-acid battery in EVs has been intensively investigated by using different approaches as summarized in [16], such as the approaches based on the impedance [17], [18], discharge current [19], [20], loaded terminal voltage [21]-[24], mathematical model [25]-[27], ampere-hour counter [28], and neural network [29], [30]. Recently, an attempt has been made to extend the approaches of impedance and neural network (NN) to BRC estimation of Ni-MH batteries in EVs. For the impedance approach in [31], a small amplitude ac signal (stimulus) is injected into the battery, and the terminal voltage (response) is measured. Then, the impedance is calculated by the ratio of the response to the stimulus. However, the impedance obtained under such condition does not include the characteristic due to the large discharge current which often occurs in the EV discharge current profile. Another impedance approach [32] is to inject a modulation current, namely a small amplitude ac signal superimposed on a large current, into the battery and then measure the terminal voltage. The impedance is deduced from the ratio of the change of terminal voltage to the amplitude of modulation current. Since the modulation current is set to be the same as the discharge current, the characteristic due to the large discharge current can be taken into account. Nevertheless, the impedance generally indicates the battery state of charge (SOC), which is actually different from the BRC in EVs. For the NN approach in [33], a NN with three layers (input, hidden, and output layers) has been applied. At the input layer, there are four neurons to represent the battery terminal voltage, discharge current, temperature, and discharged capacity. At the hidden layer, five neurons are adopted as a result of compromise between the estimation accuracy and complexity of the NN. At the output layer, there is only one neuron to indicate the BRC. The selected experimental data that reflect the features of the $\mathrm{Ni}-\mathrm{MH}$ battery are adopted to train the $\mathrm{NN}$, whereas the whole set of experimental data obtained from routine operation of $\mathrm{EVs}$ is used to verify the NN. The estimation error of this NN can be as small as 5\%. However, this NN does not take into account the influence of the EV discharge current profile on the BRC estimation. Also, to the best of the authors' knowledge, there is no analytic or semi-analytic Ni-MH circuit model available for BRC estimation in EVs.

In this paper, a new NN-based approach that can incorporate the effect of discharge current profile into the BRC estimation of Ni-MH batteries for EVs is proposed. To achieve the goal, two new concepts are introduced. First, the state of available capacity (SOAC) is defined to represent the BRC, which can be written as

$$
p(t)=1-q(t) / C_{a}
$$

where $p(t)$ denotes the SOAC, $C_{a}$ refers to the BAC at the fully charged state for a given discharge current profile of the battery, and $q(t)$ is the discharged capacity as expressed by

$$
q(t)=\int_{0}^{t} I_{d}(t) d t
$$

where $I_{d}(t)$ is the discharge current. Second, the discharged and regenerative capacity distribution is proposed to describe the discharge current profile for SOAC estimation. Consequently, a NN with the inputs of capacity distribution and temperature is newly applied to estimate the SOAC. Based on experimentation under various $\mathrm{EV}$ discharge current profiles at different temperatures, the proposed NN for SOAC estimation can be established. This $\mathrm{NN}$, which extracts the knowledge from experimental data in terms of its weights and biases, essentially represent the mapping from discharged current profiles and temperatures to the SOAC. In this sense, the forthcoming discharge current profile has been considered in the trained NN. Moreover, this NN can be easily implemented as the BRC indicator for EVs by using a low-cost microcontroller (such as the Intel 80C96). The total cost is only about a few hundred United States dollars.

Since the proposed BRC indicator is to be installed in EVs, it is too difficult to measure battery chemical parameters. As a result, all the inputs of the NN model are electrical parameters, and the proposed $\mathrm{NN}$ is an indirect way to describe chemical behaviors by using electrical parameters. Also, the BRC can be considered as a nonlinear function of multivariables, namely, the discharged and regenerative capacity at different current ranges and temperatures. However, the influence of these multivariables on the BRC is not explicit, leading to difficulty in formulating a model based on polynomial regression or multidimensional scaling techniques.

\section{EXPERIMENTATION}

The NN for SOAC estimation requires plentiful experimental data of the EV battery. To obtain these data, a battery evaluation and testing system is built at the International Research Center for Electric Vehicles, The University of Hong Kong. As shown in Fig. 1, it consists of five main parts, namely, programmable battery charger, programmable electronic load, programmable temperature chamber, power controller, and computer control and data acquisition subsystem. The resolution of data acquisition is \pm 15 bits. Based on this system, the battery can be tested under different charge and discharge currents at 


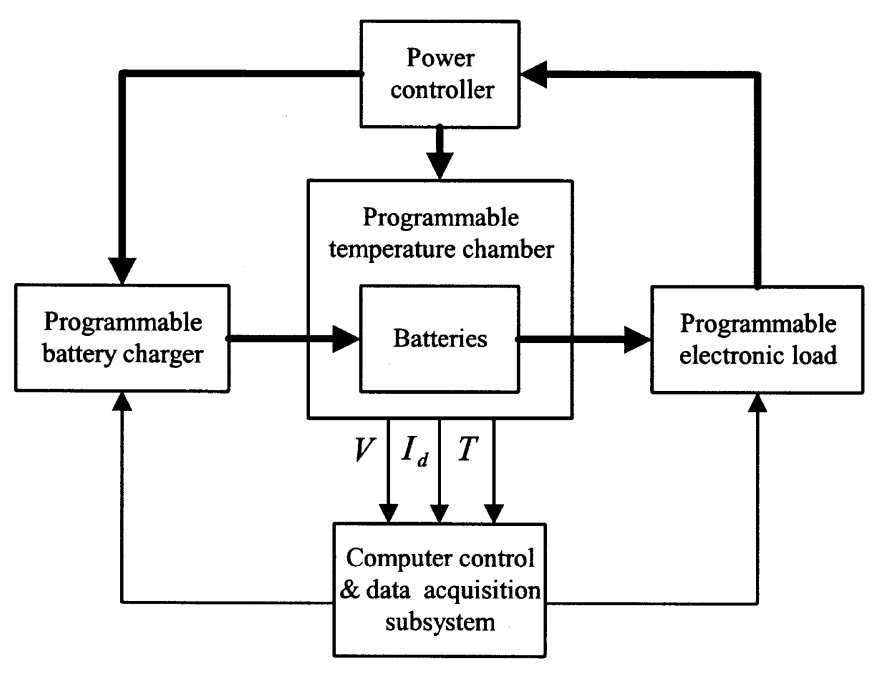

Fig. 1. Battery evaluation and testing system.

predefined temperatures. The aforementioned Ni-MH battery is adopted for this experimentation.

Since discharge current profiles strongly influence on the $\mathrm{BAC}$ and hence the EV driving range, different discharge current profiles that emulate the battery operating conditions in EVs are employed for experimentation. They correspond to the U.S. federal urban driving schedule (FUDS), U.S. federal highway driving schedule (FHDS), European reference driving cycle (ECE), and Japanese standard driving cycle (Mode 10.15). The average current of all these discharge current profiles is approximately equal to $C_{N} / 3$, where $C_{N}$ is the rated capacity of the $\mathrm{Ni}-\mathrm{MH}$ battery under investigation.

To carry out experimentation, the BAC is defined as the quantity of electricity that can be delivered at given discharge current profile and temperature until the specified cutoff voltage is reached. Mathematically, it can be written as

$$
C_{a}=\left.f\left(V(t), I_{d}(t), T(t)\right)\right|_{V(t)=V_{\text {off }}}
$$

where $V(t)$ is the battery terminal voltage, $T(t)$ is the temperature, and $V_{\text {off }}$ is the specified cutoff voltage. With different combinations of $\mathrm{EV}$ discharge current profiles and temperatures (ranging from $10^{\circ} \mathrm{C}$ to $40^{\circ} \mathrm{C}$ ), totally 32 tests are carried out. In each test, the battery at the fully charged state $(p(t)=1)$ is discharged until the specified cutoff voltage of $20 \mathrm{~V}$ is reached $(p(t)=0)$. The experimental data are automatically recorded. Fig. 2 shows four typical sets of data, namely, the FUDS, FHDS, ECE, and Mode 10.15, at the temperature of $25^{\circ} \mathrm{C}$. From the discharged capacity of each test, the corresponding BAC can readily be deduced. Then, the corresponding SOAC can be calculated by using (2).

\section{NN FOR SOAC ESTIMATION}

The essence of the NN for SOAC estimation is the relationship between the SOAC and its input parameters. These parameters should be chosen from the easily measurable ones, such as battery terminal voltage, discharge current, and temperature, as well as the easily calculated ones, such as discharged
TABLE III

LOWER AND UPPER CURRENT BOUNDS FOR CAPACITY DISTRIBUTION

\begin{tabular}{|c|c|c|c|c|}
\hline & \multicolumn{4}{|c|}{$i$} \\
\hline & 1 & 2 & 3 & 4 \\
\hline$I_{i}^{l}(\mathrm{~A})$ & 0 & $C_{N} / 2$ & $C_{N}$ & $C_{N} / 0.75$ \\
\hline$I_{i}^{u}(\mathrm{~A})$ & $C_{N} / 2$ & $C_{N}$ & $C_{N} / 0.75$ & $C_{N} / 0.5$ \\
\hline
\end{tabular}

capacity and regenerative capacity. Intuitively, the SOAC has a close relationship with the battery terminal voltage and discharge current. For this reason, they were chosen as the inputs of the NN for BRC estimation in [33]. However, the intuition is not as the case as they have been conceived. Fig. 3 illustrates the relationships between the SOAC and battery terminal voltage at a temperature of $25^{\circ} \mathrm{C}$. It can be observed that the battery terminal voltage changes considerably with fluctuating discharge current while the SOAC monotonously decreases throughout discharging process. These phenomena demonstrate that the information embedded in battery terminal voltage and discharge current can not offer a direct contribution to SOAC estimation. By comparisons, the BAC is greatly influenced by the discharge current profile. Consequently, the discharged and regenerative capacity distribution is newly proposed to describe the discharge current profile for SOAC estimation. Those discharged and regenerative capacities are obtained by integrating the currents over time, hence, offering the function of low pass filtering and inherently eliminating the effect of disturbances. Although an error may be introduced during integration of currents over time, this error causes insignificant effect on SOAC estimation because capacity distribution, not capacity, is used to represent discharge current profile for SOAC estimation. As shown in Table III, the capacity distribution based on the lower and upper current bounds of four current ranges, namely, $I_{i}^{l}$ and $I_{i}^{u}(i=1, \ldots, 4)$, is adopted. The selection of these four current ranges is to take into account the influence of discharge current levels on SOAC estimation. As a result, the proposed NN for SOAC estimation is as shown in Fig. 4.

The proposed NN consists of three layers. The first layer, namely, the input layer, has six neurons:

- $X_{1}(t)$-discharged capacity for $I_{1}^{l} \leq I_{d}(t)<I_{1}^{u}$;

- $X_{2}(t)$-discharged capacity for $I_{2}^{l} \leq I_{d}(t)<I_{2}^{u}$;

- $X_{3}(t)$-discharged capacity for $I_{3}^{l} \leq I_{d}(t)<I_{3}^{u}$;

- $X_{4}(t)$-discharged capacity for $I_{4}^{l} \leq I_{d}(t)<I_{4}^{u}$;

- $X_{5}(t)$-regenerative capacity;

- $X_{6}(t)$-temperature.

Four neurons for the discharged capacity and one for the regenerative capacity are adopted because of the following two reasons. First, the regenerative capacity is generally much lower than the discharged capacity, thus the influence of regenerative capacity on the BRC is less significant. Second, in order to reduce the complexity of the $\mathrm{NN}$, the number of neurons at the input layer should be as small as possible. The use of five neurons for the discharged and regenerative capacity can satisfy the predefined error criterion.

Considering the vector $X(t)=\left[\begin{array}{lll}X_{1}(t) & X_{2}(t) & X_{3}(t)\end{array}\right.$ $\left.X_{4}(t) X_{5}(t) X_{6}(t)\right]$, the proposed NN can be described as a function that maps the input vector $X(t)$ to the output vector 


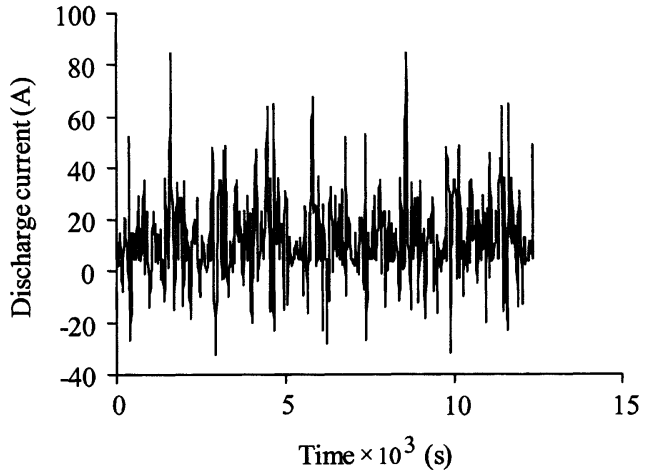

(a)

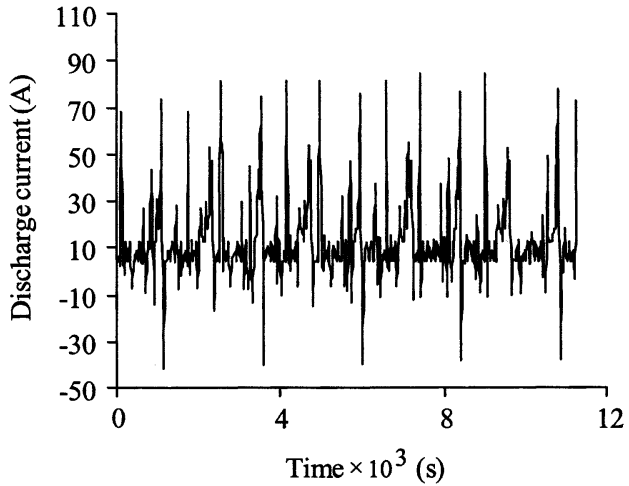

(c)

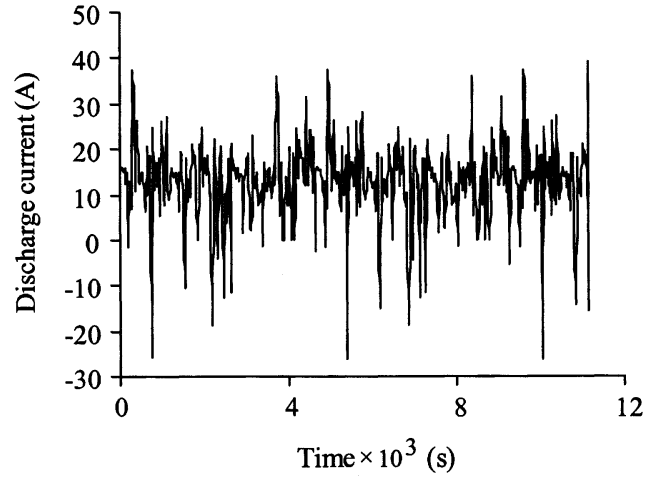

(b)

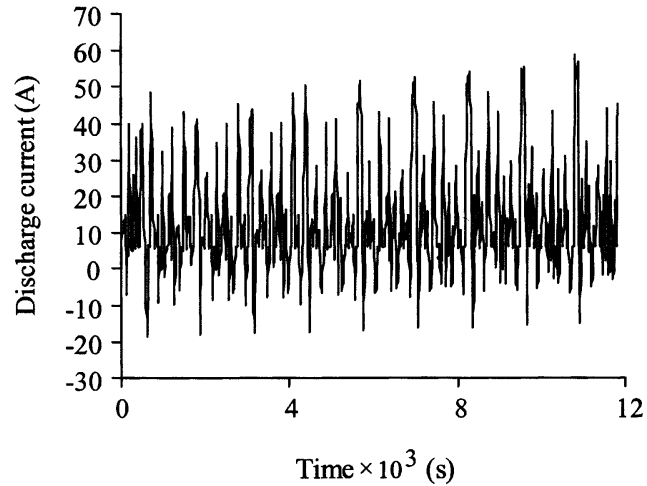

(d)

Fig. 2. Discharge current profiles of EV driving cycles. (a) FUDS. (b) FHDS. (c) ECE. (d) Mode 10.15.

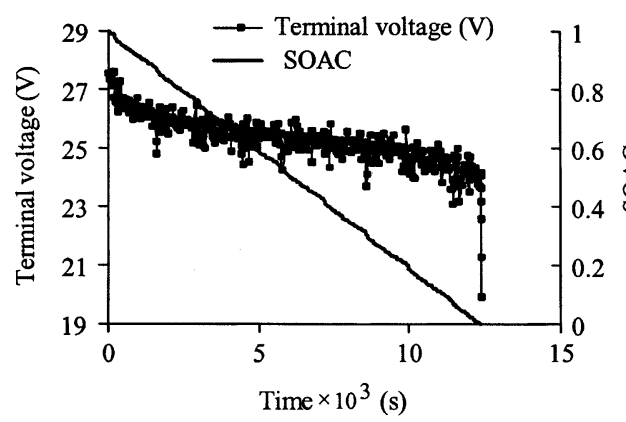

(a)

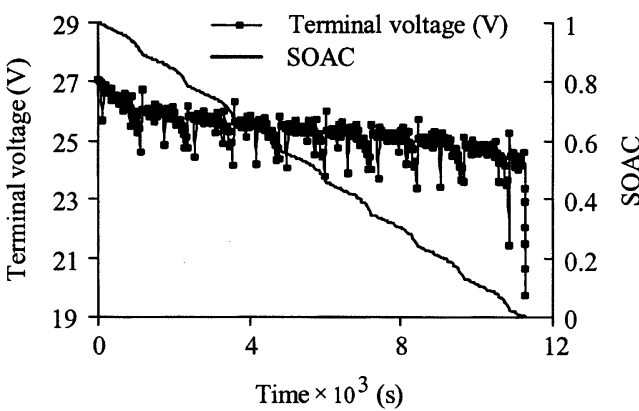

(c)

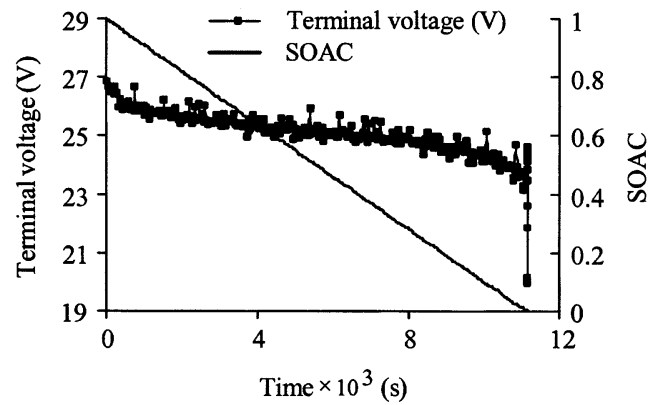

(b)

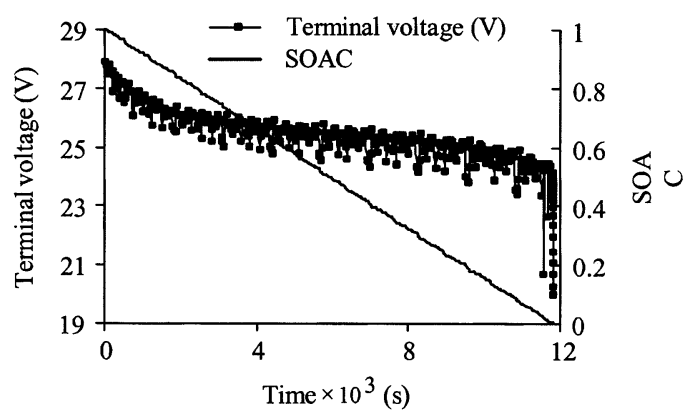

(d)

Fig. 3. Relationship between SOAC and terminal voltage under different EV discharge current profiles. (a) FUDS. (b) FHDS. (c) ECE. (d) Mode 10.15. 


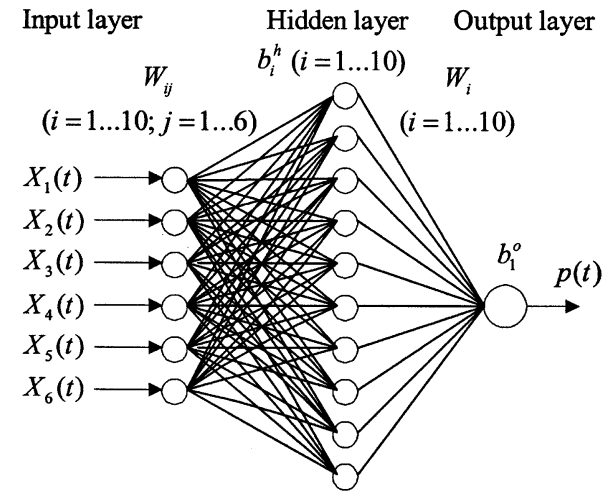

Fig. 4. Proposed NN for SOAC estimation.

$p(t)$, namely the SOAC at time $t$. Mathematically, it can be expressed as

$$
\begin{aligned}
& \widehat{p}(t)=\sum_{i}^{n} W_{i} F\left(y_{i}\right)+b_{1}^{o} \\
& F\left(y_{i}\right)=\frac{1-\exp \left(-2 y_{i}\right)}{1+\exp \left(-2 y_{i}\right)}
\end{aligned}
$$

where $\widehat{p}(t)$ represents the value of SOAC estimation, $n$ is the number of neurons at the hidden layer, $W_{i}(i=1, \ldots, n)$ are the weights between the hidden layer and output layer, $b_{1}^{o}$ is the bias at the output layer, and $y_{i}(i=1, \ldots, n)$ is the input to the $i$ th neuron at the hidden layer and is given by

$$
y_{i}=\sum_{j=1}^{6} W_{i j} X_{j}(t)+b_{i}^{h}
$$

where $W_{i j}(i=1, \ldots, n, j=1, \ldots, 6)$ are the weights between the input layer and hidden layer, and $b_{i}^{h}(i=1, \ldots, n)$ are the biases at the hidden layer. To determine the necessary number of hidden layer neurons, different numbers with $n$ ranging from 7 to 12 are examined. It can be found that the NN with 10 hidden layer neurons is preferred because there is no significant improvement in the estimation accuracy when $n$ is greater than 10.

The learning algorithm of the $\mathrm{NN}$ is a numerical process which determines the connection strength, namely, the weights between layers and the biases in neurons. During the learning process, the validation data set is incorporated to improve the generality of the NN. Based on the validation data set, the learning process is terminated when the error function begins to increase or becomes smaller than the convergence tolerance, whichever is reached first. In this paper, the convergence tolerance is set to $10^{-5}$. The error function $E$ is defined as

$$
E=\frac{1}{2} \sum_{k=1}^{m}(p(k)-\widehat{p}(k))^{2}
$$

where $m$ is the number of training data, $p(k)$ is the SOAC calculated from experimental data, and $\widehat{p}(k)$ is the SOAC estimated by the NN.

The parameters of this NN are optimized by using the Levenberg-Marquardt algorithm, which is an improved back-

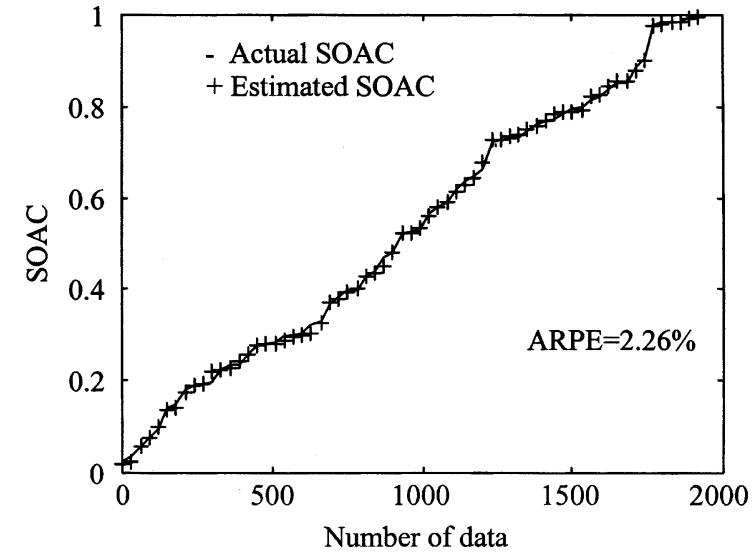

Fig. 5. Comparison between actual SOAC and estimated SOAC; based on training data.

propagation algorithm [34]. Since this algorithm is a kind of Newton's method for minimization of the sum of squares of nonlinear functions, it is well suited to minimize the error function as defined in (8). Thus, $E$ can be expressed in terms of the following parameters:

$$
H=\left\{W_{i}, b_{1}^{o}, W_{i j}, b_{j}^{h}\right\} \quad(i=1, \ldots, n, j=1, \ldots, 6) .
$$

The corresponding optimum parameters can be obtained through the following iterative process:

$$
H_{r+1}=H_{r}-A_{r}^{-1} g_{r}
$$

where $\left.A_{r} \equiv \nabla^{2} E(H)\right|_{H=H_{r}}$ and $\left.g_{r} \equiv \nabla E(H)\right|_{H=H_{r}}$ are the Hessian matrix and gradient vector of $E$ to the $r$ th iteration, respectively.

\section{RESULTS}

The data obtained from the experimentation described in Section II are used to train, validate, and verify the proposed NN. First, all data are normalized by the following equation:

$$
X_{j n}(t)=\frac{X_{j}(t)-X_{j \min }}{X_{j \max }-X_{j \min }} \quad(j=1, \ldots, 6)
$$

where $X_{j n}(t)$ is the normalized value and $X_{j \max }$ and $X_{j \min }$ are the maximum and minimum values of $X_{j}(t)$, respectively. After normalization, the whole data set, having a total of 5789 samples, is divided into three separate data sets, namely, the training, validation and testing data sets. The training data set is used to train the $\mathrm{NN}$, the validation data set is to improve the generality of the $\mathrm{NN}$, whereas the testing data set is used to verify the accuracy and effectiveness of the trained $\mathrm{NN}$ for SOAC estimation.

To assess the accuracy of SOAC estimation, the average relative percentage error (ARPE) is defined

$$
\mathrm{ARPE}=\frac{1}{N} \sum_{j=1}^{N} \frac{\left|p_{e}(j)-p_{c}(j)\right|}{\left|p_{c}(j)\right|} \times 100 \%
$$

where $N$ is the number of training or testing data, $p_{e}$ and $p_{c}$ refer to the estimated SOAC resulted from the trained NN, and the actual SOAC calculated from experimental data, respectively. Fig. 5 shows the actual SOAC and the estimated SOAC based 


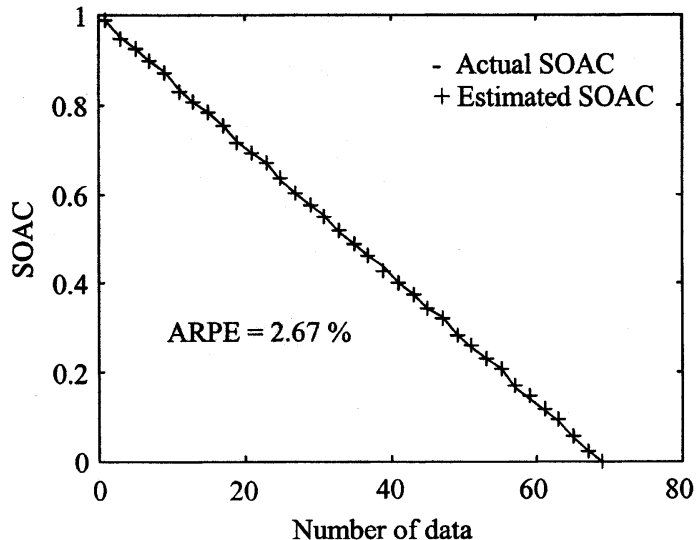

(a)

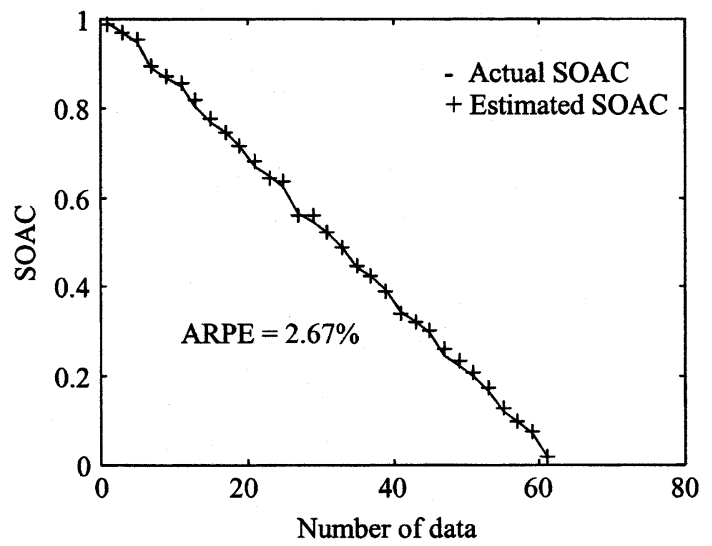

(c)

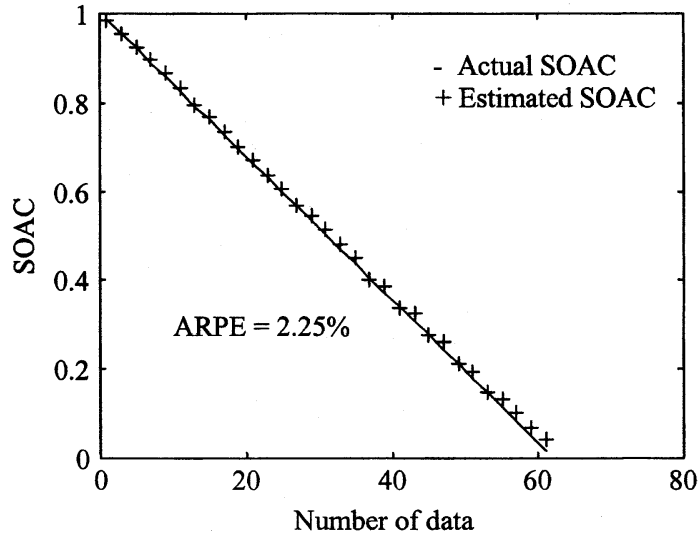

(b)

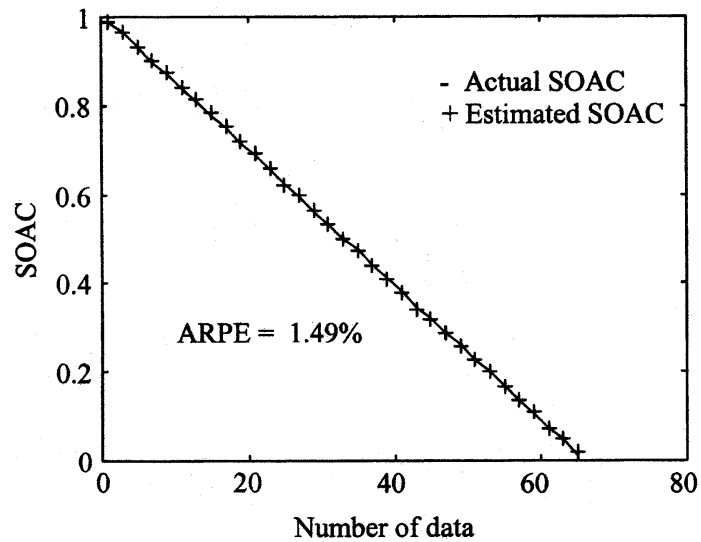

(d)

Fig. 6. Comparison between actual SOAC and estimated SOAC; based on different testing data. (a) FUDS. (b) FHDS, (c) ECE. (d) Mode 10.15.

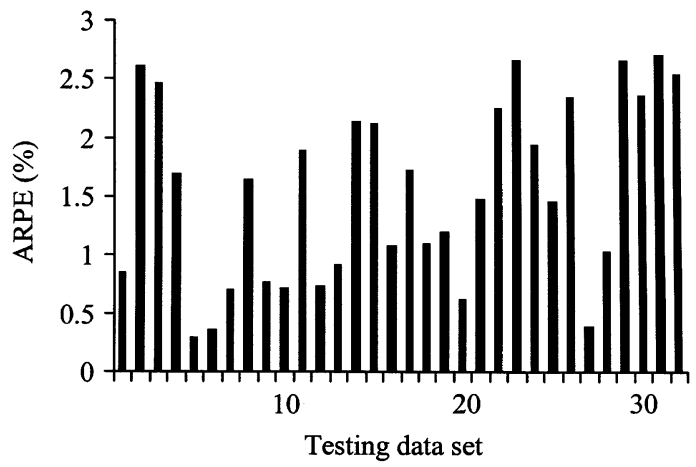

Fig. 7. Overall ARPE.

on the same training data set. As expected, the SOAC estimation is very accurate and the corresponding ARPE is only $2.26 \%$.

To testify the effectiveness of the trained NN for SOAC estimation, the testing data of each test are utilized to assess the accuracy. Fig. 6 shows a comparison between the actual SOAC and the estimated SOAC at the temperature of $25^{\circ} \mathrm{C}$ under different EV discharge current profiles. It can be found that all estimated SOACs closely agree with the corresponding actual SOACs, hence confirming that the proposed $\mathrm{NN}$ can provide accurate SOAC estimation for EVs. It should be noted that the ARPEs of all 32 tests are plotted in Fig. 7, where the maximum

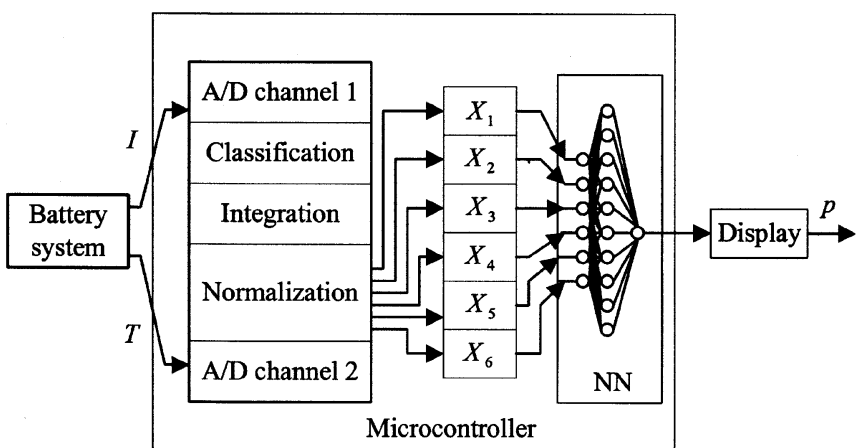

Fig. 8. Implementation of an SOAC indicator using a microcontroller.

ARPE is only 2.67\%. This demonstrates that the ARPE of the proposed NN takes an advantage over that of the NN in [33] which adopted the same battery type and was also assessed by an EV discharge current profile.

Moreover, the proposed NN can be easily implemented by a low-cost microcontroller. Fig. 8 shows the implementation diagram of a SOAC indicator based on the proposed NN. This indicator consists of four major units, namely the classification, integration, normalization and NN units. First, the classification unit is to categorize the discharge current into the predefined four current ranges as listed in Table III. Second, the integration 
unit is to integrate the discharge and regenerative currents, hence obtaining the discharged and regenerative capacity distribution. Third, the normalization unit is to normalize the capacity distribution and temperature by using (11). Finally, the NN unit is to estimate the SOAC by using (5)-(7). All these functions can be easily realized based on the assembly language embedded in the microcontroller.

\section{CONCLUSION}

In this paper, a new NN approach has been developed for BRC estimation of Ni-MH batteries in EVs. The key is to define the SOAC as the output of the NN, as well as to introduce the discharge and regenerative capacity distribution as its inputs. The SOAC can incorporate the effect of EV discharge current profiles to accurately represent the BRC of Ni-MH batteries in EVs. The discharged and regenerative capacity distribution can readily describe various discharge current profiles to adapt different EV driving cycles or sophisticated battery characteristics by easily adjusting the number of the current ranges and the corresponding upper and lower current bounds.

Comparisons between the estimated SOACs and the actual SOACs demonstrate that the proposed three-layer NN can provide accurate SOAC estimation, as the ARPEs obtained for all 32 testing data sets are less than $2.67 \%$. Moreover, this NN can be easily implemented as an on-board BRC indicator for EVs by using a low-cost microcontroller.

Further work of the proposed NN can be extended to consider the behavior of multiple battery modules and the influence of ageing effect. On the other hand, since some hybrid EVs do not allow for charging the batteries at the grid and the corresponding fully charged state is not known, the proposed NN can be further extended to those hybrid EVs. Similar to the development of dynamic models for lead-acid batteries [35], further work of the proposed NN can also be extended to the development of dynamic models for Ni-MH batteries.

\section{ACKNOWLEDGMENT}

The authors would like to acknowledge K. P. Wong and P. Ling of GP Batteries International, Ltd., in Hong Kong for their generous support.

\section{REFERENCES}

[1] C. C. Chan and K. T. Chau, "An advanced permanent magnet motor drive system for battery-powered electric vehicles," IEEE Trans. Veh. Technol., vol. 45, no. 1, pp. 1-9, Jan. 1996.

[2] _- "An overview of power electronics in electric vehicles," IEEE Trans. Ind. Electron., vol. 44, no. 1, pp. 3-13, Jan. 1997.

[3] K. L. Butler, M. Ehsani, and P. Kamath, "A matlab-based modeling and simulation package for electric and hybrid electric vehicle design," IEEE Trans. Veh. Technol., vol. 48, no. 6, pp. 1770-1778, Nov. 1999.

[4] O. Caumont, P. L. Moigne, C. Rombaut, X. Muneret, and P. Lenain, "Energy gauge for lead acid batteries in electric vehicles," IEEE Trans. Energy Convers., vol. 15, no. 3, pp. 354-360, 2000.

[5] J. Gan, K. T. Chau, C. C. Chan, and J. Z. Jiang, "A new surface-inset, permanent-magnet, brushless DC motor drive for electric vehicles," IEEE Trans. Magn., vol. 36, no. 5, pp. 3810-3818, Oct. 2000.

[6] M. Ceraol and G. Pede, "Techniques for estimating the residual range of an electric vehicle," IEEE Trans. Veh. Technol., vol. 50, no. 1, pp. 109-115, Jan. 2001.
[7] C. C. Chan, "The state of the art of electric and hybrid vehicles," Proc. IEEE, vol. 90, no. 2, pp. 247-275, 2002.

[8] S. Z. Jiang, K. T. Chau, and C. C. Chan, "Spectral analysis of a new sixphase pole-changing induction motor drive for electric vehicles," IEEE Trans. Ind. Electron., vol. 50, no. 1, pp. 123-131, 2003.

[9] K. T. Chau, Y. S. Wong, and C. C. Chan, "An overview of energy sources for electric vehicles," Energy Convers. Manage., vol. 40, no. 10, pp. 10211039, 1997.

[10] G. L. Hunt, "The great battery search [electric vehicles]," IEEE Spectrum, vol. 35, no. 11, pp. 21-28, Nov. 1998.

[11] R. C. Stempel, S. R. Ovshinsky, P. R. Gifford, and D. A. Corrigan, "Nickel metal hydride: ready to serve," IEEE Spectrum, vol. 35, no. 11, pp. 29-34, Nov. 1998.

[12] A. Paryani, "Field evaluation of Honda's EV plus battery packs," in Proc Battery Conference on Applications and Advances, 2000, pp. 83-87.

[13] K. T. Chau and Y. S. Wong, "Hybridization of energy sources in electric vehicles," Energy Convers., Manage., vol. 42, no. 9, pp. 1059-1069, 2001.

[14] I. B. Weinstock, "Recent advances in the US department of energy's energy storage technology research and development programs for hybrid electric and electric vehicles," J. Power Sources, vol. 110, no. 2, pp. 471474, 2002.

[15] D. Berndt, Maintenance-Free Batteries. Sommerset, U.K.: Research Studies, 1997, pp. 213-232.

[16] C. C. Chan, E. W. C. Lo, and W. X. Shen, "An overview of battery technology in electric vehicles," in Proc. International Electric Vehicle Symposium, 1999, CD-ROM.

[17] A. Salkind, T. Atwater, P. Singh, S. Nelatury, S. Damodar, C. Fennie, and D. Reisner, "Dynamic characterization of small lead-acid cells," J. Power Sources, vol. 96, no. 1, pp. 151-159, 2001.

[18] E. Karden, S. Buller, and R. W. De Doncker, "A frequency-domain approach to dynamical modeling of electrochemical power sources," Electrochimica Acta, vol. 47, no. 13-14, pp. 2347-2356, 2002.

[19] D. Mayer and S. Biscaglia, "Modelling and analysis of lead acid battery operation," in Proc. Telecommunications Energy Conference, vol. 2, 1989, pp. 1-6.

[20] R. Rynkiewicz, "Discharge and charge modeling of lead acid batteries," in Proc. Applied Power Electronics Conference and Exposition, vol. 2, 1999, pp. 707-710.

[21] L. Jyunichi and T. Hiroya, "Battery state-of-charge indicator for electric vehicle," in Proc. International Electric Vehicle Symposium, vol. 2, 1996, pp. 229-234.

[22] S. Matsumura, H. Higasa, M. Ohmori, A. Fukui, and T. Hayashida, "A performance test of the state-of-charge meter of lead-acid batteries," in Proc. International Electric Vehicle Symposium, vol. 2, 1996, pp. 235-238.

[23] W. X. Shen, C. C. Chan, E. W. C. Lo, and K. T. Chau, "Estimation of battery available capacity under variable discharge currents," J. Power Sources, vol. 103, no. 2, pp. 180-187, 2002.

[24] S. Sato and A. Kawamura, "A new estimation method of state of charge using terminal voltage and internal resistance for lead acid battery," in Proc. Power, vol. 2, 2002, pp. 565-570.

[25] Z. M. Salameh, M. A. Casacca, and W. A. Lynch, "A mathematical model for lead-acid batteries," IEEE Trans. Energy Convers., vol. 7, no. 1, pp. 93 98, 1992.

[26] S. Pang, J. Farrell, J. Du, and M. Barth, "Battery state-of-charge estimation," in Proc. American Control Conference, vol. 2, 2001, pp. 1644-1649.

[27] J. Marcos, A. Lago, C. M. Penalver, J. Doval, A. Nogueira, C. Castro, and J. Chamadoira, "An approach to real behaviour modeling for traction lead-acid batteries," in Proc. Power Electronics Specialists Conference, vol. 2, 2001, pp. 620-624.

[28] K. Morio, H. Kazuhiro, and P. Anil, "Battery SOC and distance to empty meter of the honda EV plus," in Proc. International Electric Vehicle Symposium, 1997, pp. 1-10.

[29] S. Malkhandi, S. K. Sinha, and K. Muthukumar, "Estimation of state of charge of lead acid battery using radial basis function," in Proc. Industrial Electronics Conference, vol. 1, 2001, pp. 131-136.

[30] A. Affanni, A. Bellini, C. Concari, G. Franceschini, E. Lorenzani, and C. Tassoni, "EV battery state of charge: Neural network based estimation," in Proc. Electric Machines and Drives Conference, vol. 2, 2002, pp. 684688.

[31] A. Salkind, C. Fennie, P. Singh, T. Atwater, and D. Reisner, "Determination of state of charge and state of health of batteries by fuzzy logic methodology," J. Power Sources, vol. 80, no. 1-2, pp. 293-300, 1999.

[32] S. Nugues, E. Rulliere, and J. P. Yonnet, "State of charge measurement by impedance spectroscopy for traction batteries," in Proc. International Electric Vehicle Symposium, vol. 2, 1996, pp. 653-658. 
[33] J. C. Peng, Y. B. Chen, R. Eberhart, and H. H. Lee, "Adaptive battery state of charge estimation using neural network," in Proc. International Electric Vehicle Symposium, 2000, CD-ROM.

[34] C. T. Lin and C. S. G. Lee, Neural Fuzzy Systems. Englewood Cliffs, NJ: Prentice-Hall, 1996, pp. 236-250.

[35] S. Barsali and M. Ceraolo, "Dynamical models of lead-acid batteries: Implementation issues," IEEE Trans. Energy Convers, vol. 17, no. 1, pp. 16-23, 2002.

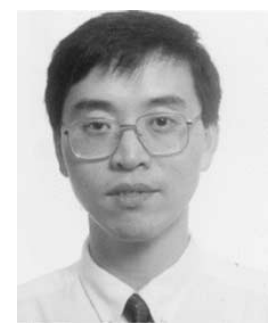

W. X. Shen (M'02) received the B.Sc. (Eng.) degree from Anhui Institute of Electrical and Mechanical Engineering, China, the M.Sc. (Eng.) degree from Shanghai Jiaotong University, China, and the Ph.D. degree from the University of Hong Kong, Hong Kong, in 1985, 1990, and 2002, respectively.

He joined the Department of Electrical Engineering at the Hefei University of Technology, China, in 1990 and has been an Associate Professor since 1995. He was a Visiting Scholar at the University of Stuttgart, Germany, from 1993 to 1994 and a Lecturer at Ngee Ann Polytechnic, Singapore, from 2002 to 2003. Currently, he works as a Lecturer at Monash University Malaysia, Malaysia. His research interests include renewable energy, electrical vehicles, power electronics, and power system.

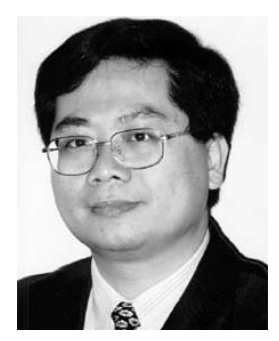

K. T. Chau (M'89-SM'04) received the B.Sc. (Eng.) with first-class honors, M.Phil., and Ph.D. degrees in electrical and electronic engineering from The University of Hong Kong, Hong Kong, in 1988, 1991, and 1993, respectively.

Currently, he works as Associate Professor in the Alma Mater, and Director of the International Research Center for Electric Vehicles. His teaching and research interests focus on three main areas: electric vehicles, electric drives, and power electronics. In these areas, he has published over 200 refereed technical papers and many industrial reports. He also co-authored a monograph "Modern Electric Vehicle Technology" published by the Oxford University Press.

Dr. Chau has served as chairs and organizing committee members for many international conferences, especially in the area of Electric Vehicles. Currently, he serves as Co-Editor of the Journal of Asian Electric Vehicles. He was selected as recipients of the Outstanding Young Researcher Award in 2003, the University Teaching Fellowship Award in 2004, and the Award for Excellence in Teaching, Learning, and Technology in 2005.

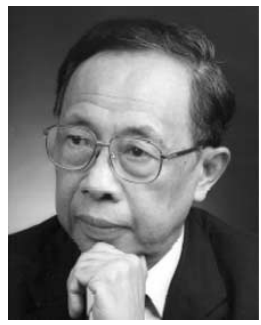

C. C. Chan (M'77-SM'77-F'92) received the B.Sc. degree from China University of Mining and Technology, Beijing, China, the M.Sc. degree from Tsinghua University, Beijing, China, and Ph.D. degree from the University of Hong Kong, Hong Kong, in 1953, 1957 , and 1981, respectively.

Currently, he is the Honorary Professor at the University of Hong Kong. He has had over 10 years industrial experience and over 30 years academic experience. He is the Founding President of the International Academy for Advanced Study, China. He has served as Visiting Professor of a number of well-known universities around the world. He is Co-founder and the Rotating President of the World Electric Vehicle Association, the President of the Asian Electric Vehicle Society, and Past President of the Hong Kong Institution of Engineers.

Prof. Chan is a Fellow of the Royal Academy of Engineering, U.K., the Chinese Academy of Engineering, the Ukraine Academy of Engineering Sciences, and the Hong Kong Academy of Engineering Sciences. He is also a Fellow of the Institution of Electrical Engineers (IEE-U.K.) and HKIE. He received the IEE International Lecture Medal in 2000 and delivered lectures on electric vehicles worldwide. He was selected as one of Asia's Best Technology Pioneers by Asiaweek in 2001, and named as "Father of Asian Electric Vehicles" by Magazine Global View.

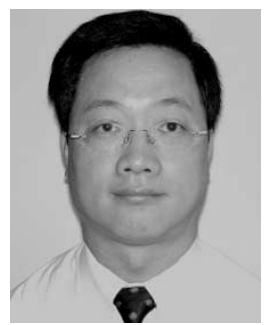

Edward W. C. Lo (M'85) received the B.Sc.(Eng.) with first-class honors, M.Phil. and Ph.D. degrees in electrical and electronic engineering from The University of Hong Kong, Hong Kong, in 1983, 1986, and 1996, respectively.

Currently, he works as an Assistant Professor in Department of Electrical Engineering in the University of Hong Kong Polytechnic University, Hong Kong. His teaching and research interests focus on three main areas: electric drive systems, power electronics, power quality, and renewable energy. In these areas, he has published over 100 refereed technical papers and many industrial reports. 